\title{
Content-Aware Selective Retransmission Scheme in Heavy Loaded Wireless Networks
}

\author{
Árpád Huszák and Sándor Imre
}

Budapest University of Technology and Economics, Department of Telecommunications, Mobile Communications and Computing Laboratory, Magyar Tudósok krt.2., H-1117 Budapest, Hungary

Tel.: +36 70514 5925, Fax: + $361463-3263$

Email: huszak@hit.bme.hu,imre@hit.bme.hu

\begin{abstract}
Streaming media is becoming increasingly prominent on the Internet, although multimedia applications have very stringent bandwidth, delay and loss requirements. In mobile environment the limited bandwidth and the higher error rate arise as an obstacle of its popularity. In many cases retransmission-based error recovery can be an attractive solution to improve the quality of the video stream, because it requires minimal network bandwidth and processing cost. In this paper we propose a content-aware selective retransmission scheme which allows the retransmission of all packets when the risk of congestion is low, but as it rises the retransmission is disabled step-by-step, but not all at once, in order of packet importance. In this work the heterogenity of H.264 streams were utilized for the determination of packet importance. The advantage of this transmitter controlled procedure is that all the needed information is available at the source due to DCCP transport protocol and its congestion control algorithm. The effectiveness of the proposed method was examined in Ns2 network simulator.
\end{abstract}

\section{Introduction}

Real-time multimedia applications are gaining prominence on the Internet. These applications are not only used in reliable wired networks but also in wireless environment where the obstacles of the expansion are the higher bit error ratio of the radio link and the limited bandwidth of the mobile links. The loss of packets in a video frames leads not only to reduce the quality of video but also results in the propagation of distortion to successive frames, because interframe-video compression algorithms such as MPEG exploit temporal correlation between frames to achieve higher compression. To minimize the end-to-end packet loss ratio the packet loss should be either prevented or subsequently handled. 
Traditional error control mechanisms generally use retransmission to provide reliability at the expense of latency. For the retransmission to be successful, retransmitted packet must arrive at the receiver in time for playback. To minimize the probability of wastefully retransmitted packets, a playout buffer is usually set up at the receiver side.

The reason of packet loss can be either the overload of the network or the bad channel conditions. In order to avoid congestion collapse of the network and to minimize the packet loss, multimedia applications may use congestion control. The performance of congestion control protocols may significantly degrade over wireless links because they confuse wireless losses with congestion losses and unnecessarily reduce the throughput. To solve this problem new congestion control were developed, like ARC [1] and WLED-ARC [2].

In this paper a selective retransmission method is introduced which efficiently manages the retransmission process in heavy loaded network. Our proposal is that we disable the retransmission of less important packets step-by-step, as the network load increases and approaches to congestion state. With disabling the retransmission of packets with specified content, we are able to control the overall stream bitrate between certain limits in order to avoid congestion. We notice that the proposed algorithm is acceptable only for pre-recorded and oneway real-time video streams, when the retransmission delay can be tolerable.

The rest of the paper is organized as follows. A review of related work in selective retransmission is presented in Section II. In Section III we introduce our content-aware selective retransmission method for multimedia applications. The obtained results are presented in Section IV. Finally, we summarize our paper and make the conclusions in the last section.

\section{Related work}

Many papers deal with QoS control, but packet losses remain inevitable because the network condition between the server and the client changes dynamically and drastically during playout. The method of these previous works can be divided to content based and network characteristic based selective retransmission algorithms.

Our work focuses on content based schemes, which basic idea is to retransmit only the important data of the bitstream, taking advantage of the motion prediction loop employed in most motion compensation based codecs. Several proposals have described retransmission schemes for recovering lost streaming packets. One of the most popular schemes, based on RTP (Real-Time Transport Protocol)[3], was proposed in [4-6]. In the scheme, when the client detects packet losses, it transmits a retransmission request packet to the server. The server then retransmits the lost packets to the client.

Feamster and Balakrishnan [7] analyzed this approach with SR-RTP [8]. This RTP extension provides semantics for requesting the retransmission of inde- 
pendently processible portions of the bitstream and a means for reassembling fragmented portions of independently processible units. They have shown that, by recovery of only the most important data in the bitstream, significant performance gains can be achieved without much additional penalty in terms of latency.

In [9] the selective retransmission of MPEG stream was analyzed with DCCP. This transport protocol provides indispensable information to apply semireliable transfer of MPEG video. The results show that the effectiveness of this protocol is considerable when selective retransmission is deployed. In [10] a semi-reliable multicast protocol is analyzed based on the IP multicast protocol and retransmission request messages. Zheng and Atiquzzaman [11] proposed a new selective retransmission scheme for multimedia transmission over noisy wireless channel using the ATM ABR service. They analyzed the system requirements and minimum receiver.

In most of the related works the receiver controls the retransmission procedure. The decision algorithm is implemented at the receiver therefore additional administration messages must be sent to the sender. Most of the prior works use NACK (Negative ACKnowledgement) or Retransmission Request messages. In our proposal no administration messages are needed because the decision procedure is located at the transmitter. The other advantage of the transmitter side decision is that the input parameters of the decision algorithm (RTT, estimated link bandwidth, etc.) are available at the source using the DCCP [12] transport protocol.

\section{Content-Aware Selective Retransmission Scheme}

Loss-tolerant real-time multimedia applications prefer UDP or UDPLite [13] but in our proposal we applied DCCP (Datagram Congestion Control Protocol) as transport protocol because it uses sequence numbering, acknowledgements and congestion control algorithms. Sequence numbers and acknowledgement are needed to identify the lost packets while the congestion control algorithms (TCP-Like Rate Control [14], TCP Friendly Rate Control [15], ARC [1], WLED-ARC [2]) manage the actual sending rate. To determinate the sending rate the congestion control algorithms estimates the round-trip-time (RTT) and the packet loss probability of the link. The packet loss probability is an important variable for our content-aware selective retransmission algorithm too.

The DCCP protocol makes it possible to identify the lost packets; therefore we can manage the retransmission of these packets. Retransmitting packets will increase the bandwidth used for the video stream transmission. The additional load due to retransmissions highly depends on the overall packet loss ratio. We can easily verify that with the increase of the packet loss probability, the needed bandwidth will be higher due to retransmissions. The expected value of the 
overall bandwidth can be calculated as follows, where $\mu$ is the video bitrate, $\mu$ ' is the additional bitrate due to retransmissions and $p$ is the loss probability:

$$
\mathrm{E}\left(\mu+\mu^{\prime}\right)=\mu+\mu p+\mu p^{2}+\ldots=\mu+\mu \frac{p}{1-p}=\mu \frac{1}{1-p}
$$

The retransmission delay can also limit the number of retransmissions if we enable multiple retransmissions, that means we allow to retransmit a lost packet when it was already retransmitted and lost. If we want to provide reliable transmission we have to allow multiple retransmissions. In other words, the number of retransmission is infinite. In this case the expected value of the delay $(d)$ due to retransmission can be calculated using the formulas of geometrical progression:

$$
\begin{gathered}
\mathrm{E}(d)=\frac{R T T}{2}(1-p)+\frac{3 R T T}{2}(1-p) p+\frac{5 R T T}{2}(1-p) p^{2}+\ldots \\
\mathrm{E}(d)=\frac{R T T}{2}(1-p) \cdot \sum_{n=0}^{\infty}(2 n+1) p^{n}=\frac{R T T}{2} \cdot \frac{1+p}{1-p}
\end{gathered}
$$

If we also consider the loss detection delay (!) the expected value of the retransmission delay is:

$$
\begin{gathered}
\mathrm{E}(d)=\frac{R T T}{2}(1-p)+\left(\frac{3 R T T}{2}+\tau\right)(1-p) p+\left(\frac{5 R T T}{2}+2 \tau\right)(1-p) p^{2}+\ldots \\
\mathrm{E}(d)=\frac{R T T}{2} \cdot \frac{1+p}{1-p}+\tau \frac{p}{1-p}
\end{gathered}
$$

In our proposed content-aware retransmission scheme we have taken only the bandwidth constrains into consideration. The estimated available bandwidth in the network is considered as the proposed sending rate of the congestion control algorithm. In order to avoid congestion the overall bandwidth should not exceed the proposed sending rate. In case when the overall video stream bitrate is higher then the calculated sending rate $\left(X_{c c}\right)$, the retransmissions must be disabled, because it makes no sense to retransmit any packet that will be lost again.

$$
\mu(t)+\mu^{\prime}(t)>X_{c c}(t) \quad \Rightarrow \quad \text { retransmission disabled }
$$

In a heavy loaded wireless network the packets should loss due to congestion and the due to bad wireless channel conditions. To efficiently estimate the available bandwidth alternative congestion control algorithm must be used. The TFRC protocol can not distinguish congestion loss and wireless loss; therefore, during wireless losses, the sending rate will be lower than the really achievable rate. Using alternative congestion control methods (ARC and WLED integrated with ARC (WLED-ARC)) which effectively estimates the congestion loss, the performance of transmission can be improved. ARC [1] is the first congestion control algorithm that models the behavior of the "ideal" TCP that doesn't react to wireless losses. ARC is a rate-control scheme that uses the following equation: 


$$
S=\frac{1}{R T T}\left(3+\sqrt{25+\frac{24}{p_{c}}}\right),
$$

where $S$ is the sending rate in packets per second and $p_{c}$ is the congestion loss probability. The latter is related to the total packet loss probability " and the wireless loss probability \# through the expression:

$$
p_{c}=\left(\frac{\pi-\omega}{1-\omega}\right)
$$

The parameter" is easily estimated from the total packets received and the total packets lost, by looking at the sequence numbers. To calculate \#, ARC relies on the MAC layer to get this loss probability. However, this approach violates the end-to-end paradigm and will not work if there is no way to obtain the wireless loss probability from lower layers.

The proposed selective retransmission scheme uses the calculated sending rate of the congestion control algorithms to decide whether to enable the retransmission of a lost packet or not. In same network conditions when the wireless loss is high, the ARC-based congestion control algorithms provide significantly higher sending rates than TFRC.

As we declared before, the retransmission must be disabled when the calculated sending rate $\left(X_{c c}\right)$ descend under the overall video bitrate $(\mu+\mu$ '). In some cases the difference between $X_{c c}$ and the video bitrate $(\mu)$ is too low to enable the retransmission of all the lost packets. In general:

$$
\mathrm{E}\left(\mu^{\prime}\right)=\mu \frac{p}{1-p}<\lambda,
$$

where $\$$ is the bound of the difference, when the retransmission of all lost packets can be enabled.

For the real-time decision algorithm:

$$
X_{c c}(t)-\mu(t)<\lambda
$$

In cases when retransmission of all lost packets is not possible, we should consider which packets should be retransmitted. Our recommendation is that the packet content must be used to select the packets for retransmission. The MPEG video frame structure gives a good opportunity to efficiently select the lost packet for retransmission. If we utilize this feature of the MPEG, we can allow the retransmission step-by-step. When the difference of the calculated sending rate and the video bitrate $\left(X_{c c}-\mu\right)$ is decreasing and not all the lost packets can be retransmitted, first the retransmission of packets that contains $\mathrm{B}$ frames must be disabled. In second step, when the difference is too small to retransmit I and P frames, $\mathrm{P}$ frame retransmission must be disabled. For the decision, the additional load due to the retransmissions should be estimated.

The extra load $\left(\mu_{I}{ }^{\prime}\right)$ due to the I frame retransmission is:

$$
\mu_{I}{ }^{\prime}=\rho_{I} \cdot p \cdot \mu,
$$


where $\%$ is the I-frames data ratio in the GOP, and can be calculated as:

$$
\rho_{I}=\frac{\text { total size of I frames in the GOP }}{\text { GOP size }}
$$

The calculation of corresponding $\mathrm{P}$ and $\mathrm{B}$ frame ratios are similar, hence $\rho_{\mathrm{I}}+\rho_{\mathrm{P}}+\rho_{\mathrm{B}}=1$. Similarly to (11) the extra loads for I+P and all frame types retransmission can be calculate as follows:

$$
\begin{gathered}
\mu_{I+P}{ }^{\prime}=\left(\rho_{I}+\rho_{P}\right) \cdot p \cdot \mu \\
\mu_{\text {all }}{ }^{\prime}=p \cdot \mu
\end{gathered}
$$

The decision process will enable or disable different frame type retransmission according to the additional load and the currently proposed congestion control sending rate. In some cases there is no possibility to determinate the current ratio, we can set the frame ratios $\rho_{\mathrm{I}}=\rho_{\mathrm{P}}=\rho_{\mathrm{B}}=0.33$ or 0.5 , but as we will see in the simulation section, there is no significant difference between the two parameter setup. The stipulation of retransmitting $\mathrm{I}, \mathrm{I}+\mathrm{P}$ and all the frames are as follows:

Table 1. Frame type retransmission

\begin{tabular}{|c|c|c|}
\hline & General ratio $\left(!_{50 \%}=\mathbf{0 . 5}\right)$ & $\begin{array}{c}\text { Ratios of the actual video } \\
\left(!_{1}, !_{P}, !_{B}\right)\end{array}$ \\
\hline \hline I frames & $\mu<X_{c c}<\mu+\mu_{50 \%}^{\prime}$ & $\mu<X_{c c}<\mu+\mu_{I}^{\prime}$ \\
\hline I+P frames & $\mu+\mu_{50 \%}^{\prime}<X_{c c}<\mu+2 \mu_{50 \%}^{\prime}$ & $\mu+\mu_{I}^{\prime}<X_{c c}<\mu+\mu_{I+P}^{\prime}$ \\
\hline all frames & $X_{c c}>\mu+2 \mu_{50 \%}^{\prime}$ & $X_{c c}>\mu+\mu_{\text {all }}^{\prime}$ \\
\hline
\end{tabular}

With the proposed method we can utilize the available bandwidth in the most efficient way, because when the bandwidth is not enough to retransmit all the lost packets, the packet selection for the retransmission is done according to packet importance. We used the calculated sending rate $\left(X_{c c}\right)$ of the ARC congestion control protocol. 


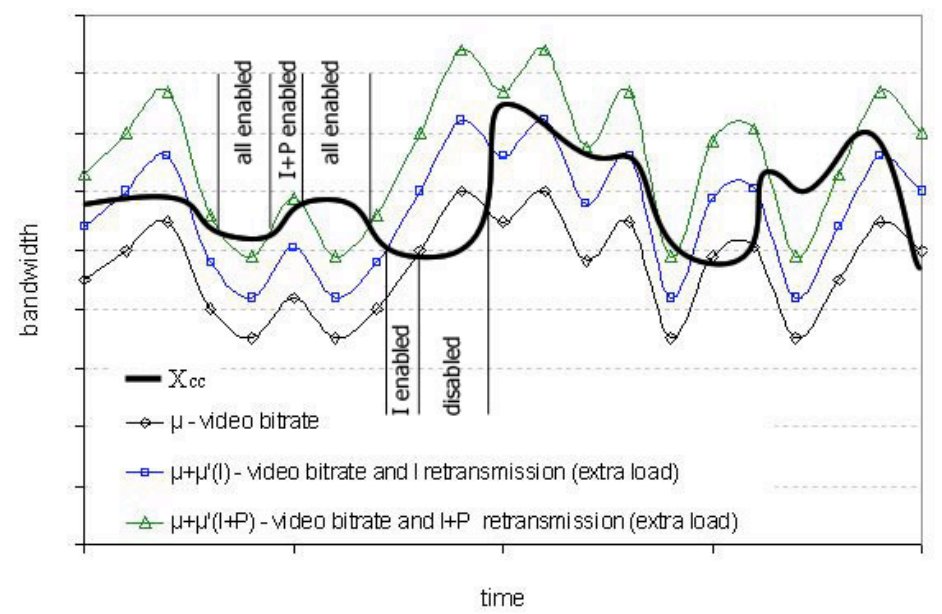

Fig. 1 The decision algorithm of the proposed content-aware retransmission method

The figure clearly illustrates the decision function. The bandwidth difference between the retransmission limits linearly depend on the overall packet loss ratio. When the packet loss is higher, the difference is becoming more and more significant. Our method can be effective when the calculated sending rate of the congestion control algorithm is similar to the video bitrate. This situation can occur when the available channel bandwidth is low like in heavy loaded networks.

\section{Simulation results}

In this section we explore the performance of the proposed ARC-based adaptive content-aware retransmission scheme in Ns2 [16]. We used dumbbell topology with $1 \mathrm{Mbps}$ links and $10 \mathrm{~ms}$ link delays. The wireless packet link was the bottleneck of the analyzed network. We have used a simple random drop model to introduce losses, with the given loss probability. In order to analyze the proposed method in heavy loaded network WWW background traffic were set up. Each WWW connection was transmitting random size of files and after the TCP transmission of the webpage, random idle interval was inserted. The idle time was determined, using exponential distribution with average value of 5 seconds, while webpage size was generated using the Pareto distribution (mean value was set to $10 \mathrm{kB}$ ). To analyze the quality of the H.264 video stream (reference video "mother and daughter"), the PSNR (Peak Signal to Noise Ratio) objective quality parameter was used. In order to emphasize the improvement of the proposed method we have generated three $\sim 155 \mathrm{kbps}$ H.264 video streams with different frame type ratios. 
Table 2. The analyzed H.264 streams

\begin{tabular}{|l|l|l|l|r|r|r|}
\hline \multirow{2}{*}{ Coding parameters } & \multirow{2}{*}{ kbps } & \multirow{2}{*}{$\begin{array}{c}\text { PSNR } \\
\text { Y [dB] }\end{array}$} & \multirow{2}{*}{ Kbps } & \multicolumn{3}{|c|}{ Frame type size ratios in the } \\
\cline { 5 - 7 } & & & & I [\%] & P [\%] & B [\%] \\
\hline \hline $\mathrm{N}=24 \mathrm{M}=3 \quad \mathrm{Q}=10$ & 159,77 & 41,24 & 159,77 & 31,93974 & 42,65924 & 25,40102 \\
\hline $\mathrm{N}=6 \quad \mathrm{M}=3 \quad \mathrm{Q}=15$ & 154,36 & 38,25 & 154,36 & 80,05087 & 10,36654 & 9,582596 \\
\hline $\mathrm{N}=6 \quad \mathrm{M}=3 \quad \mathrm{Q}=30-30-20$ & 155,07 & 31,33 & 155,07 & 12,99754 & 1,290237 & 85,71222 \\
\hline
\end{tabular}

Of course the effectiveness of the proposed content-aware retransmission method was different depending on the video stream. We have generated the video streams with very different GOP structures (N/M parameters). In the $\mathrm{N}=24 \mathrm{M}=3 \mathrm{Q}=10$ video the frame type size ratios are similar, while in the $\mathrm{N}=6$ $\mathrm{M}=3 \mathrm{Q}=15$ video, the total size of the $\mathrm{I}$ frames in the stream is really high. In case of $N=6 \quad M=3 \quad Q=30-30-20$, the $B$ frame size ratio is significant, while the total size of I and $\mathrm{P}$ frames in the stream is very low.

To achieve quality improvement successfully retransmitted packets are needed. We have made simulations with $X_{c c}$ determined by TFRC and ARC congestion control algorithm. According to the simulation results, the TFRC is not effective for our content-aware retransmission method, because the calculated sending rate $\left(X_{c c}\right)$ is too low; therefore the estimated free bandwidth $\left(X_{c c}-\mu\right)$ is not enough for the additional load due to retransmissions. When the total loss probability is low, the number of lost packet is also very low. The proposed selective retransmission method is developed for transmission in high loss probability networks. In case of high total loss probability, we should examine more possibilities: high wireless loss - high congestion loss, low wireless loss - high congestion loss and high wireless loss - low congestion loss. In all these cases the TFRC sending rate $\left(X_{c c}\right)$ is very low, but in case of ARC the sending rate will not decrease when the wireless loss is high. When the congestion loss is high we can not retransmit lost packets neither with ARC nor TFRC.

In the simulations we have analyzed the number of retransmitted packet in case of content-aware retransmission scheme and without content-aware (frame) differentiation. Selective retransmission without frame differentiation is means that the retransmission is enabled if the congestion control rate is higher then the video bitrate $\left(X_{c c}>\mu\right)$ and disable when $X_{c c}<\mu$. We have made the simulation with five different wireless loss probability setup $(0.1 \%, 1 \%, 5 \%, 10 \%, 15 \%, 20 \%)$ and twelve different background load setup (number of WWW is from 5 to 60 ). The total number of simulation tests for each video stream was 60 . In order to efficiently present the results and the overall performance of the proposed method, we have calculated the average of the measured values. 


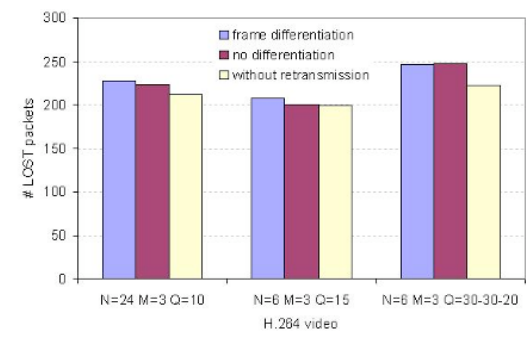

a) number of lost packets

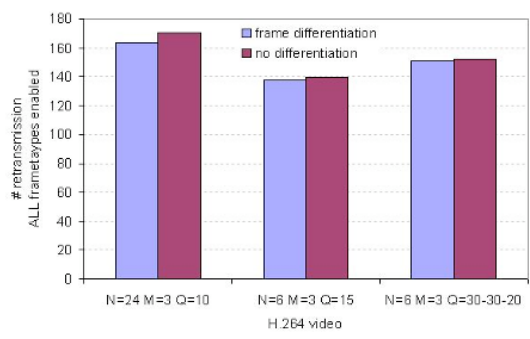

b) number of retransmitted packets,

Fig. 2 The average number of lost and retransmitted packets using the content-aware (frame differentiation) method compared with methods without content differentiation and retransmission

Transmitting more packets on the link will increase the number of lost packets too, as Fig. 2 a) shows. According to Fig. 2 b) the number of retransmitted packets, when all frame type retransmission is enabled is a little bit higher, because in case of content-aware retransmission the I and I+P frame retransmissions are not included. If we add the number of I and I+P retransmission to it, the total number of retransmissions will be very similar.

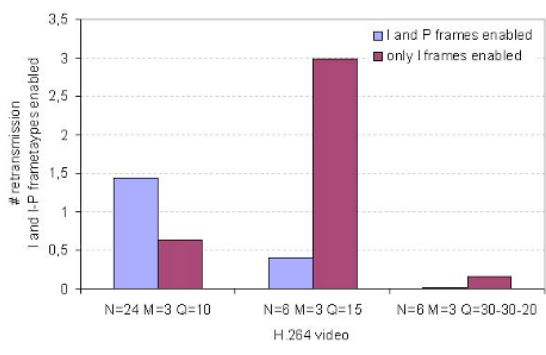

a) only I and I+P enabled

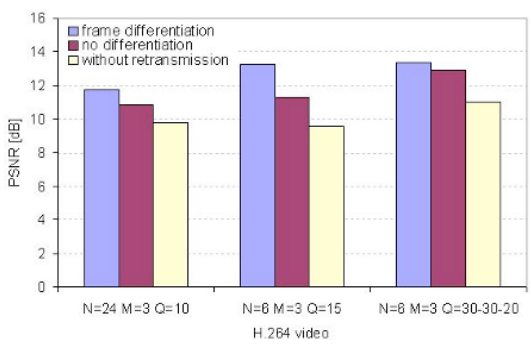

b) PSNR measurements

Fig. 3 Average number of retransmissions, when only I and I+P frame type retransmission is enabled

According to the average number retransmissions, when only I and I+P frames but no B frames were retransmitted, we were suspecting that the highest quality improvement will achieved in case of $\mathrm{N}=3 \mathrm{M}=6 \mathrm{Q}=15$ video stream. As the PSNR measurements show, the protection of I frames effectively improves the quality.

We have analyzed the PSNR improvement of the proposed retransmission scheme from the wireless loss and congestion loss point of view. In order to ef- 
ficiently illustrate the general impact of the proposed method, we have calculated the average values of the measurements. For example when the wireless loss probability was fixed, twelve measurements were done with different background traffic loads. The averages of the measured values are used in the following figures.
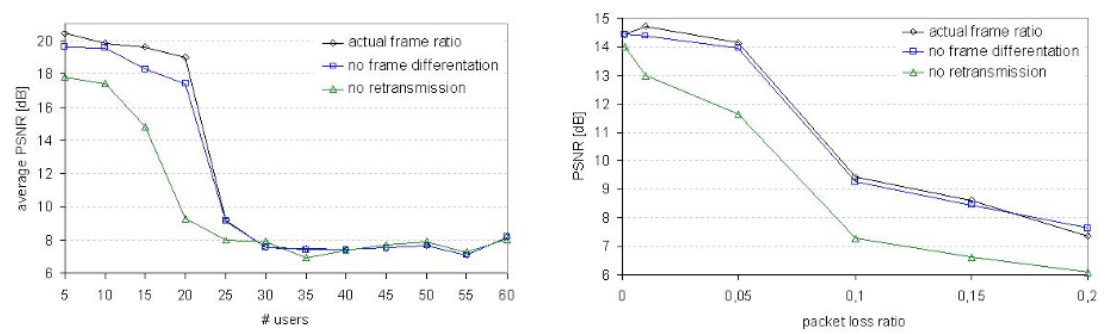

a) PSNR analysis of $\mathrm{N}=24 \mathrm{M}=3 \mathrm{Q}=10$ video stream
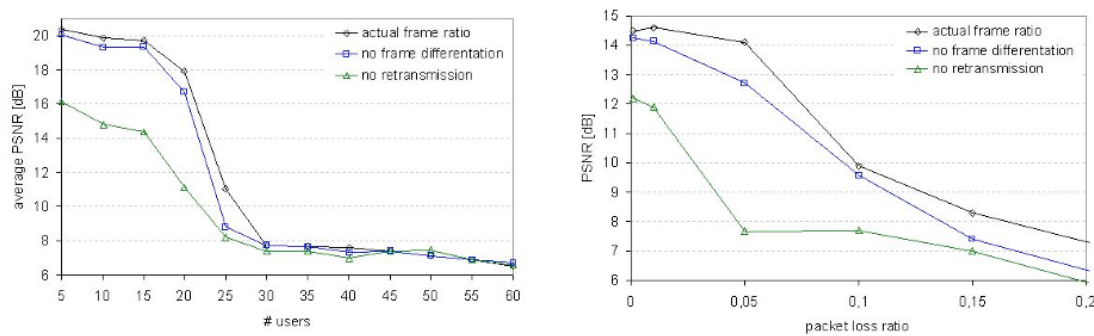

b) PSNR analysis of $\mathrm{N}=6 \mathrm{M}=3 \mathrm{Q}=15$ video stream
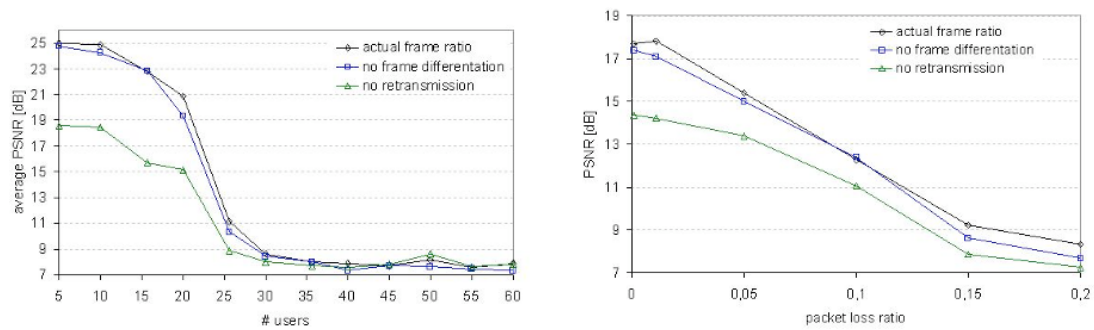

c) PSNR analysis of $\mathrm{N}=6 \mathrm{M}=3 \mathrm{Q}=30-30-20$ video stream

Fig. 4 PSNR measurements

As we can see the retransmissions can gain significant quality improvement, but using frame differentiation further PSNR improvement can be achieved. The differences in the PSNR values were not varying significantly when the packet loss was changed, while increasing the background load leads to congested state. In this state the proposed method has no effect. In the simulations $30 \mathrm{WWW}$ users were overloaded the network and made not possible to retransmit any lost packet. 


\section{Conclusion}

Bad channel conditions in wireless networks, not only degrade the video quality, but render the current congestion control algorithms that back-off on every loss. We have shown that, by recovery of the data in the bitstream considering the current state of the network, significant performance gains can be achieved without much additional penalty in terms of latency. We have integrated the ARC congestion control scheme with an adaptive re-transmission scheme in order to selectively retransmit some lost video packets. When the network utilization is very high, the extra load due to retransmissions can not be delivered. In this situation content-aware differentiation is an alternative solution to maximize the overall quality of the transmitted video stream.

\section{Acknowledgement}

This work was supported by the frame of Mobile Innovation Centre's integrated project Nr. 1.1., supported by the National Office for Research and Technology (Mobile 01/2004 contract), the Celtic-BOSS project under the framework of the EUREKA Celtic Program and the NKTH Déri Miksa project.

\section{References}

1. O. B. Akan and I. F. Akyildiz, "ARC: the analytical rate control scheme for real-time traffic in wireless networks", IEEE/ACM Transactions on Networking, vol. 12, no. 4, pp. 634-644, 2004.

2. Kamal D. Singh, David Ros, Laurent Toutain and César Viho. "Improving Multimedia Streaming over wireless using end-2-end estimation of Wireless Losses", IEEE 64th Vehicular Technology Conference, Montreal, Canada, September 2006.

3. Schulzrinne, H., Casner, S., Frederick, R., and Jacobson, V.: "RTP: A Transport Protocol for Real-Time Streaming Protocol", Internet Engineering Task Force, RFC 3550, July 2003

4. Miyazaki, A., Fukushima, H., Hata, K., Wiebke, T., Hakenberg, R., Burmeister, C., Takatori, N., Okumura, S., and Ohno, T.: "RTP Payload Formats to Enable Multiple Selective Re-transmissions", draft-ietf-avt-rtp-selret-05.txt, June 2002

5. Ott, J., Wenger, S., Sato, N., Burmeister, C., and Rey, J.: "Extended RTP Profile for RTCP-based Feedback (RTP/AVPF)", draft-ietf-avt-rtcp-feedback-05.txt, February 2003

6. Rey, J., Leon, D., Miyazaki, A., Varsa, V., and Hakenberg, R.: "RTP Retransmission Payload Format", Internet Engineering Task Force, RFC 4588, July 2006

7. M. Feamster, H. Balakrishnan, "Packet Loss Recovery for Streaming Video", 12th International Packet Video Workshop, Pittsburgh, PA, April 2002.

8. A. Miyazaki, A. H. Fukushima, K. Hata, T. Wiebke, R. Hakenberg, C. Burmeister, Matsushita, "RTP payload formats to enable multiple selective retransmission", Internet Engineering Task Force, draft-ietfavt-rtp-selret-04.txt, Nov. 2001.

9. A. Huszak, S. Imre," Selective Retransmission of MPEG Video Streams over IP Networks", CSNDSP 2006, Patras, Greece, July 2006. 


\section{Wireless and Mobile Networking}

10. Christiane Montenegro Bortoleto, Lau Cheuk Lung, Frank A. Siqueira, Alysson Neves Bessani, Joni da Silva Fraga: “A Semi-reliable Multicast Protocol for Distributed Multimedia Applications in Large Scale Networks”, MMNS 2005, LNCS 3754, Barcelona, Spain, October 24-26

11. Bing Zheng, Mohammed Atiquzzaman, "Network Requirement for Management of Multimedia over Wireless Channel”, Lecture Notes In Computer Science Vol. 2496, Proceedings of the 5th IFIP/IEEE International Conference on Management of Multimedia Networks and Services: Management of Multimedia on the Internet, London, UK, 2002.

12. Kohler, Handley, Floyd, "Datagram Congestion Control Protocol", Internet Engineering Task Force, RFC 4340, March 2006.

13. Larzon, Degermark, Pink, "The Lightweight User Datagram Protocol”, Internet Engineering Task Force, RFC 3828, July 2004.

14. S. Floyd and E. Kohler, "Profile for DCCP Congestion Control ID 2: TCP-like Congestion Control", draft-ietf-dccp-ccid2-10.txt, March 2005.

15. S. Floyd, E. Kohler, and J. Padhye, "Profile for DCCP Congestion Control ID 3: TFRC Congestion Control", draft-ietf-dccp-ccid3-11.txt, March 2005.

16. Ns-2 - Network Simulator, http:///www.isi.edu/nsnam/ns/index.html 